\title{
InterSciencePlace

\section{DIMENSÃO BIOLÓGICO-FUNCIONAL DA AVALIAÇÃO COGNITIVA DOS CONTEÚDOS DA EDUCAÇÃO FÍSICA ESCOLAR}

\section{COGNITIVE ASSESSMENT OF THE BIOLOGICAL- FUNCTIONAL DIMENSION IN SCHOOL PHYSICAL EDUCATION}

\author{
Jose Airton de Freitas Pontes Junior ${ }^{1}$, Leandro Silva Almeida ${ }^{2}$, Nicolino \\ Trompieri Filho ${ }^{3}$
}

\footnotetext{
${ }^{1}$ Faculdade de Educação da Universidade Federal do Ceará, Fortaleza-CE, Brasil, joseairton.junior@yahoo.com.br
}

\footnotetext{
${ }^{2}$ Instituto de Educação da Universidade do Minho, Braga, Portugal, leandro@ie.uminho.pt

${ }^{3}$ Faculdade de Educação da Universidade Federal do Ceará, Fortaleza-CE, Brasil, nicolino trompieri@yahoo.com.br
}

\begin{abstract}
Resumo - A escola como promotora de saúde encontra na disciplina Educação Física uma possibilidade de intervenção dos conhecimentos relacionados à atividade física e saúde no âmbito educacional. Há objetivos de aprendizagem no bloco de conteúdo Conhecimentos sobre o Corpo, porém, não há uma matriz de referência para avaliá-los cognitivamente em larga escala ao final do Ensino Fundamental. O estudo trata sobre o desenvolvimento e validação de uma matriz de referencia para avaliação cognitiva em larga escala na dimensão biológico-funcional em Educação Física escolar. A matriz foi criada baseada nos objetivos de aprendizagem cognitiva para a Educação Física no Ensino Fundamental dos Parâmetros Curriculares Nacionais. Por meio de um questionário com perguntas fechadas e 12 itens em escala, participaram do estudo 232 professores e
\end{abstract}


pesquisadores em Educação Física no Ensino Fundamental (6ำ ao $9^{\circ}$ ano) das 5 regiões do Brasil, sendo $57,3 \%$ mestres e doutores e $42,7 \%$ graduados e especialistas. Realizou-se análise fatorial exploratória por meio do programa SPSS v.22.0. Os resultados indicam que: 1) a amostra foi adequada ao estudo ( $\left.\mathrm{KMO}=0,936 ; p \leq 0,01 ; \mathrm{X}^{2}=1787,154 ; \mathrm{gl}=66\right)$, 2 ) os 12 itens possuem explicação satisfatória (Comunalidades $\geq 0,5) ; 3$ ) satisfatória variância total explicada $(64,9 \%) ; 4) 2$ fatores (Cargas fatoriais de 0,564 a 871), 5) alta consistência interna ( $\alpha=0,9$; Ritc de 0,592 a 843). Consideramos que a matriz de referência é adequada para auxiliar os professores e pesquisadores a elaborarem instrumentos de avaliação cognitiva em larga escala em relação aos conteúdos na dimensão biológicofuncional da Educação Física no Ensino Fundamental.

Palavras-chave: Educação Física escolar. Avaliação cognitiva. Saúde.

\begin{abstract}
The school as a health proponent encounters through Physical Education a possibility of integration of knowledge related to physical activity and health in the education field. There are learning objectives in the content section of Knowledge about the Body; however, no reference matrix exists to cognitively evaluate them on a large scale at the end of elementary school. The study focuses on the development and validation of a reference matrix for large scale cognitive assessment of the biological - functional dimension in Physical Education. The matrix was created based on cognitive learning objectives of the National Curriculum Parameters for Physical Education in Elementary Education. Through a questionnaire with closed questions and 12 items on scale, 232 teachers and researchers in Physical Education in Primary School (6th to 9th grade) from 5 regions of Brazil participated in the study, with $57,3 \%$ of them having masters and doctorates and $42,7 \%$ being graduates and specialists. We conducted exploratory factor analysis using SPSS v.22.0 software. The results indicate the following: 1) the sample was adequate to the study $\left.\left(\mathrm{KMO}=0,936 ; \mathrm{p} \leq 0,01 ; \mathrm{X}^{2}=1787,154 ; \mathrm{gl}=66\right), 2\right)$ the 12 items have satisfactory explanation (commonalities $\geq 0,5$ ), 3 ) satisfactory total variance explained $(64,9 \%), 4)$ factors (factor loadings from 0,564 to 871 ), 5) high internal consistency ( $\alpha=0,9$; Ritc 0,592 to 843 ). We consider the reference matrix suitable for use by teachers and researchers to help them develop cognitive assessment tools for large scale evaluation in relation to the contents of the biological- functional dimension of Physical Education in Elementary Education.
\end{abstract}

Keywords: School Physical Education. Cognitve assessment. Health.

\title{
1. Introdução
}

Nossa sociedade, que há décadas buscava emergencialmente controlar as doenças causadas por bactérias e vírus, hoje, perde milhares de vidas por doenças ligadas 
ao estilo de vida sedentário e consumo inadequado de alimentos. As doenças crônicas não transmissíveis como o diabetes mellitus, hipertensão e outras atingem boa parte da população acima de 40 anos e já se tornam freqüentes em jovens adultos, adolescentes e crianças (PITANGA, 2002; OPAS/OMS, 2003). Por serem de difícil controle farmacológico, estratégias de estilo de vida ativo têm sido sugeridas como alternativa para a prevenção e o tratamento dessas patologias (GUEDES et al, 2002; NAHAS, 2003; GUISELINI, 2004).

Na Pesquisa Nacional de Amostra por Domicílio (PNAD) de 2008, Knuth et al (2011) analisaram os dados sobre a prática de atividade física que evidenciam a presença do sedentarismo em mais de $20 \%$ da população, a diminuição do deslocamento ativo com o aumento da escolaridade e a grande quantidade de horas diárias frente a televisão. Conclusões similares decorrem do estudo de Hallal et al (2010), com os dados da Pesquisa Nacional de Saúde do Escolar, apontando a prevalência de baixa atividade física em adolescentes brasileiros, principalmente entre as meninas. Ainda nesse estudo, a maioria dos participantes apresentavam uma grande quantidade de tempo frente à televisão e a baixa prevalência de participação nas aulas de Educação Física na semana anterior à pesquisa.

O período da adolescência tem sido caracterizado como um importante momento na perspectiva de melhora da saúde (GUEDES et al, 2002). Este momento compreende a etapa da vida que vai dos 12 ou 13 anos até aproximadamente os 19 anos de idade, assumindo-se como fase de transição entre a infância e a idade adulta (COLE; COLE, 2004). A adolescência se caracteriza como uma fase de instabilidade e conflito emocional provocados pela maturação biológica (puberdade) e psicológica. Além dos aspectos biológico e psicológico, a adolescência também se caracteriza como fenômeno social, pois nesse período o sujeito assume mais responsabilidades, participa de novos grupos sociais e adquire uma maior autonomia em relação à família (BOCK, 2007). Vale lembrar que essas características são válidas para as sociedades ocidentais do começo do século XXI, podendo não caracterizar outras sociedades com culturas diferentes (COLE; COLE, 2004).

Nesta fase da vida, o adolescente passa também por mudança no âmbito escolar. Geralmente o adolescente está concluindo o Ensino Fundamental e 
ingressando no Ensino Médio (DARIDO, 1999). Esta etapa do sistema de ensino, equivalente à última fase da Educação Básica, tem como finalidade o aprofundamento dos conhecimentos adquiridos no Ensino Fundamental, bem como a formação do cidadão para a vida social e para o mercado de trabalho (CORREA; MORO, 2004). Nalguns países este nível escolar exige do adoelscente a escolha de vias de formação distintas de acordo com as suas capacidades cognitivas e motivações, iniciando o adolescente a pensar num curso e/ou profissão e na sua identidade profissional.

Dentre as disciplinas escolares, a Educação Física é um importante meio de promoção da saúde e de prevenção de doenças (GUEDES, 1999; NAHAS, 2003). Apesar disso, a Educação Física escolar durante muito tempo, e ainda hoje, é vista como uma atividade recreativa e esportiva. Segundo Ferreira (2001, p.45), "para que os alunos adotem estilos de vida ativa e tenham autonomia para a prática do exercício, é necessário que os outros conteúdos sejam desenvolvidos na educação física escolar". Apesar desta consciencialização, a Educação Física escolar permanece como uma atividade eminentemente prática e as atividades propostas nas aulas de muitas escolas não possuem vínculo com a proposta pedagógica da instituição ou sequer com uma proposta educativa (CORREA; MORO, 2004).

Hoje, várias abordagens convivem entre si, sendo a Educação Física relacionada aos esportes uma das que mais se destaca. No entanto, a importância da atividade física para a saúde vem sendo discutida na escola por possibilitar a prevenção de doenças, por meio de uma Educação Física relacionada à saúde (FERREIRA, 2001). Esta perspectiva, aplicada às escolas, objetiva estimular os alunos a terem um embasamento sobre a prática da atividade física, uma consciência do seu corpo e alimentação, um pensamento crítico sobre o que é saudável ou maléfico para a saúde, além de evitar doenças ligadas à modernidade (estresse, câncer ou obesidade, entre outras).

A Educação Física, em diversos momentos de sua história, apresentou um importante papel na aquisição da saúde, independentemente desta ser voltada para a guerra ou para a vida cotidiana. $\mathrm{Na}$ escola, a atividade física não necessariamente é bem executada a fim de propiciar esse benefício. Esportes e jogos de competição não são especificamente considerados saúde, visto que, até mesmo, podem agravar 
o bem estar dos alunos. Essas atividades, quando não bem conduzidas pelo professor, envolvem a exclusão, acabando por não valorizarem o companheirismo e o aspecto lúdico do esporte. Por exemplo, o esporte que deveria privilegiar temas diversificados e capazes de influenciar numa formação para a vida em sociedade de forma mais humana, solidária e participativa, vê-se reduzido à transmissão de técnicas e táticas, regras institucionalizadas, alta competitividade, sem contextualização e desvinculado do seu real compromisso com a educação. Da forma com que vem sendo desenvolvido, o esporte não tem propiciado a socialização e a produção de conhecimentos por parte dos alunos, realizando apenas a prática pela prática, o fazer pelo fazer e a reprodução para a adaptação. Segundo Ferreira (2001, p.45), "para que os jovens pratiquem desporto e atividade física regularmente não basta que dominem técnicas e regras. É preciso que saibam como realizar tais atividades com segurança e eficiência".

A escola, como espaço de educação em geral e de intervenção social, deve consolidar a promoção da saúde como um objetivo de todos os grupos que a compõem. Através da partilha destes ideais entre professores, alunos, gestores e comunidade, deve ocorrer uma proposta que preze pela busca da saúde da coletividade. Essa prática da educação em saúde deve ser reforçada pela ação social através de redes sociais que privilegiem a prática da atividade física, capacitando o sujeito para cuidar de si e agir pela defesa da saúde do grupo (BRASIL, 1998). Aliás, é interessante apontar que hoje a população em geral mostra ter informação, o que nem sempre significa conhecimento, sobre saúde, qualidade de vida, exercícios físicos, esportes, nutrição, doenças e dietas da modernidade. Contudo a falta de um saber técnico, a educação das atitudes e comportamentos deveria fazer pensar na função educativa da escola, pois, ao mesmo tempo, assistimos a taxas crescentes de obesidade infantil, hipertensão e cardiopatias em adolescentes e jovens adultos, causados, principalmente, pela ansiedade e 0 estresse do dia-dia moderno, além de insônia, depressão ou diabetes, dentre outras. Daí o fazer todo o sentido Nahas (2003, p.152), ao afirmar que "quando os alunos atingem 14-16 anos de idade (transição para o Ensino Médio) é preciso enfatizar os conteúdos sobre atividade física para aptidão física e saúde".

A perspectiva deste artigo é discutir a Educação Física na sua vertente ligada 
à saúde e bem-estar, afirmando a necessidade de se abordar temas teóricos e práticos nas aulas, devendo ser uma educação direcionada ao esclarecimento da saúde da população. Este movimento pode rentabilizar a emergência desde o final da década de 80 de uma geração que valoriza como nunca a importância do movimento e da atividade física, os hábitos saudáveis, o trato adequado e higiênico do corpo, a importância de se alimentar bem e o esclarecimento sobre o impacto positivo destas atitudes no corpo e na promoção da saúde.

Para uma discussão mais completa do papel da disciplina de Educação Física na formação dos adolescentes, torna-se importante dispor de instrumentos de avaliação cognitiva que possibilitem identificar o nível de conhecimento dos alunos sobre os conteúdos que são lecionados. Para tanto, o objetivo deste estudo centrase no desenvolvimento e validação de uma matriz de referência para avaliação cognitiva em larga escala dos conteúdos da Educação Física no Ensino Fundamental ( $9^{\circ}$ ano) na dimensão biológico-funcional da atividade física.

\section{Método}

O estudo é do tipo exploratório, transversal e de abordagem quantitativa que contou com a participação professores e pesquisadores brasileiros que trabalhavam e/ou pesquisavam sobre Educação Física no Ensino Fundamental (6ำ ao $9^{\circ}$ ano). Como critério de inclusão, consideramos os professores e pesquisadores que trabalharam pelo menos há um ano na faixa de ensino citada e/ou tivessem experiências acadêmicas diversas sobre o tema, tais como publicação de trabalhos científicos, docência no ensino superior, projeto de extensão, dentre outros. Como critérios de exclusão, não utilizamos nesse trabalho os docentes que não eram graduados em Educação Física, nem se encontravam a cursar a graduação nesta área.

\subsection{Amostra}

Participaram do estudo 232 professores e pesquisadores em Educação Física no Ensino Fundamental (6ำ ao $9^{\circ}$ ano) das 5 regiões do Brasil, sendo $57,3 \%$ mestres e 
doutores e $42,7 \%$ graduados e especialistas. A maioria $(66,4 \%)$ era do sexo masculino, com média de 36,9 anos de idade e média 7,2 anos de atuação na faixa de ensino citada anteriormente. A coleta de dados ocorreu por meio de uma plataforma eletrônica em que foram enviados convites para o correio eletrônico de mais de 1500 professores e pesquisadores em Educação Física escolar no Brasil. Todos os participantes foram voluntários e confirmaram o Termo de Consentimento Livre e Esclarecido (TCLE) a distancia, via plataforma, inserindo nome e endereço do correio eletrônico pessoal.

\subsection{Instrumento}

Utilizou-se um questionário contendo itens agrupados em escalas ou dimensões, a fim de coletar o nível de concordância dos professores e pesquisadores sobre os descritores para avaliação cognitiva em larga escala dos conteúdos lecionados na disciplina de Educação Física referente ao $9^{\circ}$ ano do Ensino Fundamental. Mais concretamente neste artigo reportam-se os itens agrupados na dimensão biológicofuncional da atividade física da Educação Física Os itens foram elaborados baseados nos objetivos de aprendizagem e nos conteúdos do bloco Conhecimentos sobre o Corpo da Educação Física no Ensino Fundamental dos Parâmetros Curriculares Nacionais (BRASIL, 1998).

\subsection{Procedimentos}

Para a análise dos dados, realizamos distribuição de frequências, média e coeficiente de variação, bem como análise dos fatores extraídos via análise fatorial exploratória pelo método das Componentes Principais. Para essas análises utilizouse programa IBM SPSS, versão 22.0. 


\section{Resultados}

Com o objetivo de identificarmos os fatores latentes (variáveis não-observadas) que estão relacionados aos conteúdos sobre o Conhecimento sobre o Corpo (BRASIL, 1998), elencamos os descritores ou itens desta dimensão do questionário (variáveis observadas). Foi observado que o estudo apresentou amostra adequada (KMO = 0,$936 ; p \leq 0,01 ; x^{2}=1787,154 ; g l=66$ ) e com os valores das comunalidades dos itens superiores a 0,5 , que indica que os itens apresentam satisfatória explicação (HAIR et al, 2005). A análise fatorial realizada recorreu ao método das Componentes principais sendo retidos dois fatores. O segundo aproxima-se do critério de Kaiser que fixa o auto-valor igual ou acima da unidade, sendo importante a sua retenção pois consegue explicar acima de $5 \%$ da variância dos itens, o que para Maroco (2011) deve ser considerado no momento de extração dos fatores. Na Tabela 1 apresentamos as saturações dos itens nos dois fatores, bem como a sua percentagem de variância explicada na base dos dois fatores isolados (comunalidades).

Tabela 1 - Comunalidades e cargas fatorias extraídas dos componentes

\begin{tabular}{llll}
\hline & & \multicolumn{1}{l}{ Componentes } \\
\hline & Comunalidades & 1 \\
\hline $\begin{array}{l}\text { D6 - Identificar exemplos de atividades físicas } \\
\text { relacionadas à força e resistência muscular }\end{array}$ &, 620 &, 871 \\
$\begin{array}{l}\text { D5 - Identificar exemplos de atividades físicas } \\
\text { relacionadas à flexibilidade }\end{array}$ &, 562 &, 844 \\
$\begin{array}{l}\text { D4 - Identificar exemplos de atividades físicas } \\
\text { relacionadas à capacidade cardiorrespiratória }\end{array}$ &, 811 &, 814 \\
$\begin{array}{l}\text { D2 - Diferenciar atividade física aeróbia de anaeróbia } \\
\text { D3 - Estabelecer relações entre os resultados de um }\end{array}$ &, 795 &, 715 \\
teste físico e o impacto para a saúde &, 850 &, 642 \\
D8 - Calcular o Índice de Massa Corpórea &, 633 &, 564 \\
\hline $\begin{array}{l}\text { D11 - Reconhecer os efeitos das atividades físicas no } \\
\text { corpo }\end{array}$ &, 563 &
\end{tabular}


D7 - Inferir sobre os efeitos da atividade física regular sobre a qualidade de vida

D19 - Estabelecer relações entre nutrição e desempenho físico

D12 - Identificar os efeitos do esporte de rendimento na saúde dos atletas

D10 - Estabelecer relações entre atividade física e as doenças cronicos-degenerativas

D14 - Identificar os componentes da aptidão física relacionada à saúde

Como podemos observar, os dois fatores extraídos explicam $64,9 \%$ da variância dos 12 itens, estando seis itens agrupados por cada fator. Maroco (2011) sugere que $50 \%$ de explicação é suficientes para considerar que os itens representam a estrutura em análise, embora outros autores aconselhem um valor não inferior a $60 \%$ (HAIR et al, 2005). Por outro lado, verificamos que a saturação dos itens no respectivo fator não desce abaixo de 0,56 , deixando antever bons índices de consistência interna dos itens em cada fator (alfas de Cronbach superiores a 0,80 ).

No Fator 1 encontram-se os itens dos conteúdos dos componentes da aptidão física relacionada à saúde, daí designarmos por Aptidão física relacionada à saúde. Os itens que apresentaram maiores médias de concordância para os docentes e pesquisadores investigados foram D2 - Diferenciar atividade física aeróbia de anaeróbia, D5 - Identificar exemplos de atividades físicas relacionadas à flexibilidade e D8 - Calcular o Índice de Massa Corpórea. Esse fator obteve consistência interna de $\alpha=0,91$, significância de $p \leq 0,01$ para o teste $T$ de Hotelling, com valores da correlação item $x$ total corrigida (Ritc) acima de 0,66 e o alfa permanece suficiente mesmo ao eliminar cada item (TABELA 2). 
Tabela 2 - Análise descritiva dos itens do Fator 1

\begin{tabular}{|c|c|c|c|c|c|}
\hline & $\begin{array}{l}\text { Média } \\
\text { (escala } \\
0 \text { a } 5 \text { ) }\end{array}$ & $\begin{array}{l}\text { Desvio } \\
\text { Padrão }\end{array}$ & CV\% & Ritc & $\begin{array}{l}\text { Alfa se o item } \\
\text { for eliminado }\end{array}$ \\
\hline $\begin{array}{l}\text { D2 - Diferenciar atividade física } \\
\text { aeróbia de anaeróbia }\end{array}$ & 3,56 & 1,072 & 30,1 & ,700 & ,899 \\
\hline $\begin{array}{l}\text { D3 - Estabelecer relações entre os } \\
\text { resultados de um teste físico e o } \\
\text { impacto para a saúde }\end{array}$ & 3,13 & 1,059 & 33,9 & 665 & ,904 \\
\hline $\begin{array}{l}\text { D4 - Identificar exemplos de } \\
\text { atividades físicas relacionadas à } \\
\text { capacidade cardiorrespiratória }\end{array}$ & 3,38 & 1,062 & 31,4 & 832 & ,879 \\
\hline $\begin{array}{l}\text { D5 - Identificar exemplos de } \\
\text { atividades físicas relacionadas à } \\
\text { flexibilidade }\end{array}$ & 3,50 & ,967 & 27,6 & ,798 & ,885 \\
\hline $\begin{array}{l}\text { D6 - Identificar exemplos de } \\
\text { atividades físicas relacionadas à } \\
\text { força e resistência muscular }\end{array}$ & 3,35 & 995 & 29,7 & 843 & 879 \\
\hline $\begin{array}{l}\text { D8 - Calcular o Índice de Massa } \\
\text { Corpórea }\end{array}$ & 3,36 & 1,096 & 32,6 & 659 & ,905 \\
\hline
\end{tabular}

A Educação Física, como ressalta Nahas (2003, p.159), deve "Propiciar a aquisição de conhecimentos sobre atividade física para o bem estar e a saúde em todas as idades". Na pesquisa de Silveira e Silva (2011), a maioria dos participantes $(60 \%)$ acertaram os itens que tratavam sobre o conhecimento de atividade física na prevenção de várias doenças, assim como nos resultados de Barros e Silva que indicam que mais de $50 \%$ dos adolescentes participantes da pesquisa tiveram resultados semelhantes no nível de conhecimento. Balbinotti e colaboradores (2011), afirmam que os adolescentes "parecem ter consciência da importância da atividade física regular para o combate à obesidade" (2011, p.391), pois, em seu estudo, a saúde foi o principal motivo para a prática de atividade física para os adolescentes. Já para Copetti e colaboradores (2013) propõe ações interdisciplinares em Educação Física e Ciências, já que há temas relacionados as duas áreas que podem ser trabalhados de forma diversificada e ampla. Ainda nesse 
estudo, os autores apontam que a faixa de Ensino Básico carecer de ações educativas e formativas sobre atividade física e saúde, mesmo os adolescentes apresentando bons bom nível de conhecimento entre esse assunto e a prevenção de doenças e bem-estar, pois, para os autores, os adolescentes conhecem o assunto, mas não praticam.

O Fator 2 apresenta descritores sobre os aspectos relacionados com as interpretações dos impactos da atividade física na saúde, podendo por isso designar-se Impactos da atividade física para a saúde. Os itens com maiores níveis de concordância foram D7 - Inferir sobre os efeitos da atividade física regular sobre a qualidade de vida e D11 - Reconhecer os efeitos das atividades físicas no corpo. A consistência interna obtida foi de $\alpha=0,86$, significância de $p \leq 0,01$ para o teste $T$ de Hotelling, com valores da correlação item $x$ total corrigida (Ritc) acima de 0,592 e o Alfa permanece suficiente mesmo ao eliminar cada item (Tabela 3).

Tabela 3 - Análise descritiva dos itens do Fator 2

\begin{tabular}{lccccc}
\hline & $\begin{array}{c}\text { Média } \\
\text { (escala } \\
\text { 0 a 5) }\end{array}$ & $\begin{array}{l}\text { Desvio } \\
\text { Padrão }\end{array}$ & CV\% & Ritc & $\begin{array}{c}\text { Alfa se o item } \\
\text { for eliminado }\end{array}$ \\
\hline $\begin{array}{l}\text { D7 - Inferir sobre os efeitos da } \\
\text { atividade física regular sobre a } \\
\text { qualidade de vida }\end{array}$ & 3,89 & 1,070 & 27,5 &, 664 &, 833 \\
\hline $\begin{array}{l}\text { D10 - Estabelecer relações entre } \\
\text { atividade física e as doenças } \\
\text { crônicas não transmissíveis }\end{array}$ & 3,31 & 1,198 & 36,1 &, 690 &, 828 \\
\hline $\begin{array}{l}\text { D11 - Reconhecer os efeitos das } \\
\text { atividades físicas no corpo }\end{array}$ & 3,82 &, 994 & 26,0 &, 716 &, 825 \\
\hline $\begin{array}{l}\text { D12 - Identificar os efeitos do } \\
\text { esporte de rendimento na saúde } \\
\text { dos atletas }\end{array}$ & 3,28 & 1,164 & 35,5 &, 582 &, 849 \\
\hline $\begin{array}{l}\text { D14 - Identificar os componentes } \\
\text { da aptidão física relacionada à } \\
\text { saúde }\end{array}$ & 3,19 &, 984 & 30,8 &, 673 &, 832 \\
\hline $\begin{array}{l}\text { D19 - Estabelecer relações entre } \\
\text { nutrição e desempenho físico }\end{array}$ & 3,07 & 1,077 & 35,1 &, 592 &, 846 \\
\hline
\end{tabular}


A importância do conhecimento sobre saúde e seus impactos no estilo de vida ativo e prevenção de doenças é evidenciado em vários estudos. Serafim, Jesus e Pierin (2010) indicam que o conhecimento sobre os hábitos de vida saudáveis é um fator determinante para o controle da hipertensão em adultos. No entanto, no estudo de Mendes e colaboradores (2010), com sujeitos acima de 20 anos, a principal fonte de informação sobre a prática de atividade física foi a televisão, seguindo pelo professor de Educação Física e o médico. Por sua vez, Souza e colaboradores (2011) analisando os resultados de programas de intervenção que tinham por objetivos potencializar mudanças no estilo de vida alimentar e de atividade física de escolares, verificaram que a dimensão educacional e de informação estavam presentes nas atividades propostas, por exemplo, palestras, cartazes, atividades práticas e teóricas, dramatização, dentre outros. Os resultados evidenciam o sucesso desses programas nos objetivos traçados e a relação da dimensão educacional para atingi-los.

Ferreira, Oliveira e Sampaio (2013) apontam que os professores de Educação Física, participantes do estudo, se limitam a percepção de saúde aos aspectos de ausência de doenças. No mesmo estudo, há relatos que de que o tema saúde é relacionado mais com as aulas teóricas. No entanto, para Pereira (2011) as aulas teóricas e práticas devem contextualizar os conteúdos da saúde as dimensões cognitivas, procedimentais e atitudinais.

Para auxiliar nessa abordagem da saúde, Rufino e Darido (2013) relatam a pesquisa realizada com alunos na faixa de 12 anos sobre a utilização do livro didático como recurso para tematizar os vários aspectos relacionados a saúde e as práticas corporais. Os resultados, baseados nas falas dos participantes do estudo, que haviam temas interessantes e que o livro foi atrativo para as discussões. A partir do momento que o professor de Educação Física passa a orientar seus estudantes sobre a prática das atividades físicas, sobre os conceitos de saúde e potencializar a formação crítica dos alunos, provavelmente os adolescentes terão autonomia e senso crítico questionador sobre as informações que receberem e as atitudes a serem tomadas.

Finalmente, já numa lógica de validade de conteúdo do questionário, apreciando a concordância dos pesquisados sobre avaliação cognitiva dos 
conteúdos relacionados à dimensão biológico-funcional da Educação Física, o Fator 1, numa escala de 0 a 10, obteve nota média de 6,76 (com CV\% = 25,59) e o Fator 2, com 6,87 (com CV\% = 24,20). Percebemos pelas médias obtidas dos fatores que os docentes e pesquisadores que participaram do estudo possuem maior nível de concordância com o fator Impactos da atividade física à saúde. Mesmo assim, os índices obtidos são claramente positivos e valorizam o questionário validado no quadro dos objetivos de avaliação a que se propõe. Assim, na Tabela 4 descrevemos os itens referentes aos conteúdos da Educação Física para o Ensino Fundamental (9ªno) na dimensão biológico-funcional da atividade física tomando os dois temas em que subdivide.

Tabela 4 - Matriz de referencia na dimensão Biológico-funcional da Atividade Física

\begin{tabular}{|c|c|}
\hline \multicolumn{2}{|r|}{ DIMENSÃO II - BIOLÓGICO-FUNCIONAL DA ATIVIDADE FISICA } \\
\hline Temas & Descritores \\
\hline $\begin{array}{l}\text { Aptidão física } \\
\text { relacionada à } \\
\text { saúde }\end{array}$ & $\begin{array}{l}\text { D2 - Diferenciar atividade física aeróbia de anaeróbia } \\
\text { D3 - Estabelecer relações entre os resultados de um teste físico e o impacto para } \\
\text { a saúde } \\
\text { D4 - Identificar exemplos de atividades físicas relacionadas à capacidade } \\
\text { cardiorrespiratória } \\
\text { D5 - Identificar exemplos de atividades físicas relacionadas à flexibilidade } \\
\text { D6 - Identificar exemplos de atividades físicas relacionadas à força e resistência } \\
\text { muscular } \\
\text { D8 - Calcular o Índice de Massa Corpórea }\end{array}$ \\
\hline $\begin{array}{l}\text { Impactos da } \\
\text { atividade física } \\
\text { à saúde }\end{array}$ & $\begin{array}{l}\text { D7 - Inferir sobre os efeitos da atividade física regular sobre a qualidade de vida } \\
\text { D10 - Estabelecer relações entre atividade física e as doenças crônico- } \\
\text { degenerativas } \\
\text { D11 - Reconhecer os efeitos das atividades físicas no corpo } \\
\text { D12 - Identificar os efeitos do esporte de rendimento na saúde dos atletas } \\
\text { D14 - Identificar os componentes da aptidão física relacionada à saúde } \\
\text { D19 - Estabelecer relações entre nutrição e desempenho físico }\end{array}$ \\
\hline
\end{tabular}




\section{Conclusão}

A pesquisa apresentou a validação de um conjunto de descritores (itens) para a avaliação cognitiva em larga escala dos conteúdos da Educação Física no Ensino Fundamental na dimensão biológico-funcional da atividade física. Os dois fatores ou temas extraídos foram: Fator 1 - Aptidão física relacionada à saúde e Fator 2 Impactos da atividade física à saúde. O resultados indicam, ainda, que o Fator 1 apresentam maior percentual de variância explicada, mas o Fator 2 tem maior nível de concordância dos pesquisados.

Face aos valores obtidos a propósito da validade interna e de conteúdo, assim como da consistência interna dos itens para os dois fatores identificados, podemos assumir que o instrumento agora validado pode contribuir para novos estudos na área e, inclusive, pode servir de referência para a elaboração de instrumentos de avaliação do nível de conhecimento sobre os conteúdos da atividade física e saúde. Igualmente, a par do diagnóstico dos conhecimentos dos alunos, o instrumento pode servir para avaliar o real impacto das aprendizagens ou de programas de intervenção educacional no campo da atividade física e sua relevância para a área da saúde.

\section{Referências}

BALBINOTTI, M. A. A., ZAMBONATO, F., BARBOSA, M. L. L., SALDANHA, R. P., BALBINOTTI, C. A. A. Motivação à prática regular de atividades físicas e esportivas: um estudo comparativo entre estudantes com sobrepeso, obesos e eutróficos. Motriz, Rio Claro, v.17 n.3, p.384-394, jul./set. 2011.

BARROS, F. C., SILVA, M. C. Conhecimento sobre atividade física e fatores associados em adolescentes estudantes do ensino médio da zona rural. Revista Brasileira de Atividade Física \& Saúde, Pelotas/RS, v. 18, n.5, p.594-603, Set/2013.

BOCK, A. M. B. Adolescência como construção social: estudo sobre livros destinados a pais e educadores. Revista Semestral da Associação Brasileira de Psicologia Escolar e Educacional (ABRAPEE), Campinas, v.11, n.1, p.63-76, jan./jun.2007. 
BRASIL, Ministério da Educação e Cultura. Parâmetros Curriculares Nacionais Educação Física. Brasília: MEC/SEF, 1998.

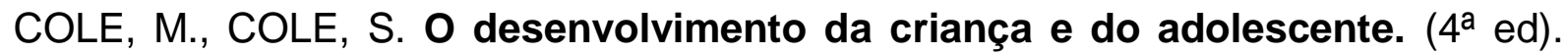
Porto Alegre: Artmed, 2004.

COPETTI, J., SOARES, R. G., LARA, S., LANES, K. G., PUNTEL, R. L., FOLMER, $V$. Conhecimento de adolescentes sobre saúde e fatores de risco para doenças e agravos não transmissíveis: sugestão de abordagem interdisciplinar. Revista Ciências \& Ideias. v. 4, n.2, jul 2013

CORREA, I. L. S., MORO, R. L. Educação física escolar: reflexão e ação curricular. ljuí: Editora Unijuí, 2004.

DARIDO, S. C. Educação física no ensino médio: reflexões e ações. Revista Motriz, Rio Claro/SP, v.5, n.2, p.138-145, dez.1999.

FERREIRA, H. S., OLIVEIRA, B. N., SAMPAIO, J. J. C. Análise da percepção dos professores de Educação Física acerca da interface entre a saúde e a Educação Física escolar: conceitos e metodologias. Revista Brasileira de Ciências do Esporte, Florianópolis, v. 35, n. 3, p. 673-685, jul./set. 2013

FERREIRA, M. S. Aptidão física e saúde na educação física escolar: ampliando o enfoque. Revista Brasileira de Ciências do Esporte, Campinas, v.22, n.2, p.41-54, jan.2001.

GUEDES, D. P. Educação para a saúde mediante programas de educação física escolar. Revista Motriz, Rio Claro/SP, v.5, n.1, jun.1999.

GUEDES, D. P. et al. Aptidão física relacionada à saúde e fatores de risco predisponentes às doenças cardiovasculares em adolescentes. Revista Portuguesa de Ciências do Desporto, Porto, v.2, n.5, p.31-46, 2002.

GUISELINI, M. Aptidão física, saúde e bem-estar. São Paulo: Phorte Editora, 2004.

HAIR, J. F., ANDERSON, R. E., TATHAM, R. L. T., ; BLACK, W. C. Análise multivariada de dados. (5 $5^{\mathrm{a}}$ ed). Porto Alegre, RS: Bookman, 2005.

HALlAL, P. C., KNUTH, A. G., CRUZ, D. K. L., MENDES, M. I., MALTA, D. C. Prática de atividade física em adolescentes brasileiros. Ciência \& Saúde Coletiva, v. 15 (Supl. 2), p.3035-3042, 2010.

KNUTH, A. G., MALTA, D. C., DUMITH, S. C., PEREIRA, C. A., MORAES NETO, O. L., TEMPORÃO, J. G., PENNA, G., HALLAL, P. C. Prática de atividade física e sedentarismo em brasileiros: resultados da Pesquisa Nacional por Amostra de Domicílios (PNAD) - 2008. Ciência \& Saúde Coletiva, v. 16, n. 9, p.36973705, 2011 
MAROCO, J. P. Análise Estatística com o SPSS Statistics. (5ª ed). Pêro Pinheiro: ReportNumber, 2011.

MENDES, M. A., ROMBALDI, A. J., AZEVEDO, M R., BIELEMANN, R. M., HALLAL, $P$. C. Fontes de informação sobre a importância da atividade física: estudo de base populacional. Revista Brasileira de Atividade Física \& Saúde, v. 15, n. 3, 2010.

NAHAS, M. Atividade física, saúde e qualidade de vida. ( $3^{\underline{a}}$ ed). Londrina: Midiograf, 2003.

PEREIRA, F. M. Procedimentos de ensino de conteúdos cognitivos em aulas de Educação Física escolar. Revista BioMotriz. n. 5, nov. 2011.

PITANGA, F. J. G. Epidemiologia, Atividade Física e Saúde. Revista Brasileira Ciência e Movimento. v. 10 n. 3 p. 49-54, Brasília: julho, 2002.

OMS/OPAS, Organização Pan-Americana da Saúde. Doenças crônicodegenerativas e obesidade: estratégia mundial sobre alimentação saudável, atividade física e saúde. Brasília, 2003.

RUFINO, L. G. B., DARIDO, S. C. Educação física escolar, tema transversal, saúde e livro didático: possíveis relações durante a prática pedagógica. Revista Brasileira Ciência e Movimento, v.21, n.3, p.21-34, 2013.

SERAFIM, T. S., JESUS, E. S., PIERIN, A. M. G. Influência do conhecimento sobre o estilo de vida saudável no controle de pessoas hipertensas. Acta Paul Enfermagem, v.23, n.5, p.658-64, 2010.

SILVEIRA, E. F., SILVA, M. C. Conhecimento sobre atividade física dos estudantes de uma cidade do sul do Brasil. Motriz, Rio Claro, v.17 n.3, p.456467, jul./set. 2011.

SOUZA, E. A., BARBOSA FILHO, V. C., NOGUEIRA, J. A. D., AZEVEDO JR, M. R. Atividade física e alimentação saudável em escolares brasileiros: revisão de programas de intervenção. Caderno de Saúde Pública, Rio de Janeiro, v.27, n.8, p.1459-1471, ago, 2011.

\section{Sobre os autores}

Jose Airton de Freitas Pontes Junior - Aluno de doutorado em Educação Brasileira da Universidade Federal do Ceará (UFC). Bolsista PDSE/CAPES - Proc. no11512/13-0. Pesquisador-estudante do Núcleo de Avaliação Educacional 
(NAVE/CNPq).

Leandro Silva Almeida - Doutor em Psicologia pela Universidade do Porto. Professor Catedrático do Departamento de Psicologia do Instituto de Educação e Psicologia da Universidade do Minho, Portugal.

Nicolino Trompieri Filho - Doutor em Educação Brasileira pela Universidade Federal do Ceará (UFC). Professor do Programa de Pós-Graduação em Educação Brasileira da UFC. Pesquisador do Núcleo de Avaliação Educacional (NAVE/CNPq). 